\title{
Characterization of a Fiber-Coupled 36-Core 3-Mode Photonic Lantern Spatial Multiplexer
}

Rommel, Simon; Mendinueta, José Manuel Delgado; Klaus, Werner; Sakaguchi, Jun; Olmos, Juan Jose Vegas; Awaji, Yoshinari; Tafur Monroy, Idelfonso ; Wada, Naoya

Published in:

Photonic Networks and Devices 2017

Link to article, DOI:

10.1364/NETWORKS.2017.NeW3B.2

Publication date:

2017

Document Version

Peer reviewed version

Link back to DTU Orbit

Citation (APA):

Rommel, S., Mendinueta, J. M. D., Klaus, W., Sakaguchi, J., Olmos, J. J. V., Awaji, Y., Tafur Monroy, I., \& Wada, N. (2017). Characterization of a Fiber-Coupled 36-Core 3-Mode Photonic Lantern Spatial Multiplexer. In Photonic Networks and Devices 2017 [NeW3B.2] Optical Society of America (OSA).

https://doi.org/10.1364/NETWORKS.2017.NeW3B.2

\section{General rights}

Copyright and moral rights for the publications made accessible in the public portal are retained by the authors and/or other copyright owners and it is a condition of accessing publications that users recognise and abide by the legal requirements associated with these rights.

- Users may download and print one copy of any publication from the public portal for the purpose of private study or research.

- You may not further distribute the material or use it for any profit-making activity or commercial gain

- You may freely distribute the URL identifying the publication in the public portal 


\title{
Characterization of a Fiber-Coupled 36-Core 3-Mode Photonic Lantern Spatial Multiplexer
}

\author{
Simon Rommel ${ }^{1,2, *}$, José Manuel Delgado Mendinueta ${ }^{1}$, Werner Klaus ${ }^{1}$, \\ Jun Sakaguchi $^{1}$, Juan José Vegas Olmos ${ }^{3}$, Yoshinari Awaji ${ }^{1}$, Idelfonso Tafur Monroy ${ }^{2}$ \\ and Naoya Wada ${ }^{1}$ \\ ${ }^{1}$ Photonic Network System Laboratory, National Institute of Communication and Information Technology (NICT), \\ 4-2-1 Nukui-Kitamachi, Koganei, Tokyo 184-8795, Japan \\ ${ }^{2}$ Department of Photonics Engineering, Technical University of Denmark, 2800 Kgs. Lyngby, Denmark \\ ${ }^{3}$ Mellanox Technologies, Ledreborg Allé 130B, 4000 Roskilde, Denmark \\ *sirem@fotonik.dtu.dk
}

\begin{abstract}
A fiber-coupled 108-port photonic lantern spatial-MUX is characterized with a spatially-diverse optical vector network analyzer. Insertion loss, mode-dependent losses, and time response are measured, showing significant mode mixing at a fiber splice.
\end{abstract}

OCIS codes: 060.4230 Multiplexing, 120.3180 Interferometry, 060.4510 Optical communications.

\section{Introduction}

Space division multiplexing (SDM) using fibers with multiple cores and/or supporting multiple modes has enabled increasing the capacity of optical communication networks beyond that of conventional single mode fibers [1,2]. Fewmode fibers (FMFs) supporting multiple spatial modes allow transmission of a number of channels on an orthogonal combination of modes of the FMF, at the cost of requiring multiple-input multiple-output (MIMO) equalization to remove the mode mixing and scrambling occurring in the fiber and during mode (de-)multiplexing [2-4]. Multi-core fibers (MCFs) reduce the footprint and increase capacity at the cost of introducing inter-core crosstalk [1].

Spatial multiplexers (SMUX) are an essential component in SDM systems and their properties directly impact system performance. For the MIMO equalizer to recover the original signals despite significant mode-mixing and resulting signal scrambling across modes in FMFs, mode-dependent loss (MDL) must be small [3]. Furthermore, mode scrambling at the transmitter improves tolerance to MDL and system capacity is maximized if all modes supported by the fiber are used to transmit information [3]. Thus, an SMUX should have low insertion loss (IL) to preserve optical power and low MDL to maintain MIMO processing capability, and should excite all the available orthogonal modes.

In this paper, a 36-core few-mode spatial multiplexer SMUX similar to [5], consisting of 36 photonic lanterns supporting three modes each, is characterized by measuring the full impulse response of all 36 photonic lanterns pertaining to the 36 different cores using a spatially-diverse optical vector network analyzer (SDM-OVNA). The measurement is performed in a reflective manner, with the SMUX coupled to $2.9 \mathrm{~m}$ of a 36-core 3-mode MCF, including one splice and cleaved at the fiber end. IL and MDL for each photonic lantern are calculated and their impulse response is analyzed. The MCF cores were designed with high differential mode delay (DMD) [2] and as a result allows identification of the different mode groups within the time domain response, showing significant coupling between mode groups at the 3-mode MCF splice.

\section{Spatially-Diverse Optical Vector Network Analyzer}

Swept-wavelength interferometry allows measurement of the complex transfer function $H(\omega)$ of an optical device under test (DUT) [6]. To measure the full complex matrix of a multiport SDM component, the scheme is extended to an SDM-OVNA by introducing different delays to separate the transfer matrix components of different spatial paths $[4,7]$. The SDM-OVNA setup, shown in Fig. 1, measures the full $6 \times 6$ transfer matrix of one of the photonic lanterns in the 36-core few-mode SMUX and consisted of a continuously swept laser, followed by an interferometer where one arm serves as a reference while the other arm includes the DUT. To simultaneously measure the impulse response of the DUT in both polarizations and on all three spatial modes of the photonic lantern, first the swept signal is split and one arm delayed before combining them with orthogonal polarizations; second, the resulting signal was split and different delays induced before applying the signal to the input ports of the DUT. As the measurement was performed in a 


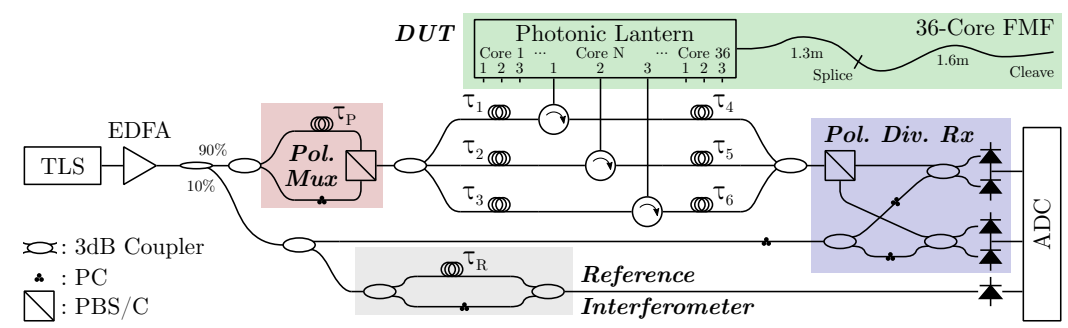

Fig. 1. Spatially-diverse optical vector network analyzer setup for characterization of the 36-core three-mode photonic lantern; TLS: continuously swept tunable laser, EDFA: erbium doped fiber amplifier, PC: polarization controller, PBS/C: polarization beam splitter/combiner, ADC: analog to digital conversion.

reflective manner, optical circulators were used to separate the reflected light and another set of delays was applied before combining the reflections into a single fiber. The resulting signal, together with the output of the reference arm, was detected by a polarization diversity receiver and the interference fringes stored on a digital sampling oscilloscope. An additional reference interferometer allowed compensation of sweep frequency non-linearity $[4,6]$.

The inclusion of the delay $\tau_{\mathrm{P}}$ between the two input polarizations and the delays $\tau_{1}$ to $\tau_{6}$ before and after the DUT ensures the components $h_{i j}(t)$ of the time-domain transfer matrix appear well separated in the inverse Fourier transform $h(t)$ of the received interferogram and can be extracted by simple temporal windowing. The Fourier transform of each $h_{i j}(t)$ yields $H_{i j}(\omega)$ and thus allows reconstruction of $H(\omega)$. Finally, IL and MDL can be calculated from the singular values of $H(\omega)$.

\section{Characterization of 36-Core Few-Mode Photonic Lantern}

The measurement of the 36 photonic lanterns of the SMUX yields a $6 \times 6$ time domain impulse response for each core as shown in Fig. 2(a). Each $2 \times 2$ sub-matrix corresponds to the four possible polarization paths between an input-output pair and summation over the latter gives a $3 \times 3$ matrix as shown in Figs. 2(c) and (d). In the latter, significantly different behavior is observed, with either five or three temporal peaks visible in each $h_{i j}(t)$. Similar behavior was found for cores located in opposite halves of the fiber as indicated on the camera image of the cleaved fiber facet in Fig. 2(b), with cores in the upper-right half predominantly showing all five peaks present, while cores in the lower-left show only three peaks; cores between the highlighted regions, including the central core, show five peaks for some input-output combinations while for others only three peaks are present. In both cases, the presence of multiple peaks suggests the photonic lantern excites both mode groups, although with different efficiency, resulting in varying peak amplitudes.

The fiber attached to the SMUX supports two mode groups with two modes in the $\mathrm{LP}_{01}$ group and four modes in the $\mathrm{LP}_{11}$ group [2] and by design has high DMD on the order of $7 \mathrm{ps} / \mathrm{m}$. Assuming negligible DMD within each mode group and taking the mode-mixing occurring at discrete points (i.e. the photonic lantern, the splice and the fiber facet) to be dominant over mode-mixing along the fiber-as is evident by the presence of well separated peaks-the possible mode-mixing paths are as shown in Fig. 2(e); the lower half of the tree is symmetric to the upper, with the resulting $\Sigma \Delta \tau$ reduced by $\Delta \tau_{1}$. With a DMD of $7 \mathrm{ps} / \mathrm{m}$ and fiber lengths of approximately $1.3 \mathrm{~m}$ and $1.6 \mathrm{~m}$ before and after the splice, this yields a total of nine different expected relative delays as shown below the curves in Fig. 2(f), forming five distinct delay groups with uniform spacing.

The outer peaks in the impulse response correspond to the case where a mode excited by the photonic lantern undergoes no mode mixing and thus their delays allow direct estimation of the DMD of the fiber, with DMDs between $6.5 \mathrm{ps} / \mathrm{m}$ and $7.5 \mathrm{ps} / \mathrm{m}$ found for the different cores, corresponding well with previous measurements [2]. The locations of the three inner peaks match the calculated delay groups, as is shown for two cores in Fig. 2(f). The different delays within each group are not resolved, due to insufficient measurement resolution resulting from laser phase noise. The presence of more than two peaks suggests significant mode-mixing to always be present at the splice, although with mixing characteristics differing between cores, resulting in different numbers of peaks; mode-mixing at the fiber facet cannot be further analyzed as the delays within each delay group are collapsed.

Finally, IL and MDL are calculated from the reconstructed transfer matrix, with IL normalized using a reference trace to exclude losses in the measurement system and the loss arising from the reflection at the cleaved facet. Fig. 3(a) shows IL averaged over the wavelength range of the sweep, finding a mean IL of $8.8 \mathrm{~dB}$. The calculated wavelength-averaged MDL values range between $12.6 \mathrm{~dB}$ and $17.4 \mathrm{~dB}$, with a mean of $14.9 \mathrm{~dB}$ as shown in Fig. 3(b). 
(a)

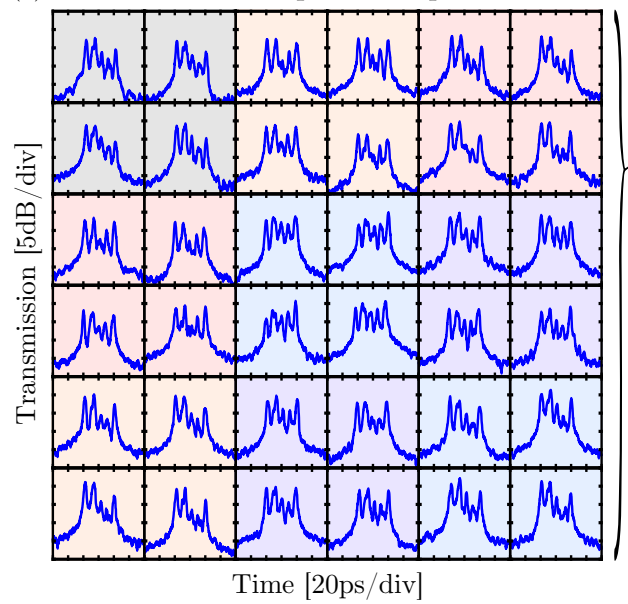

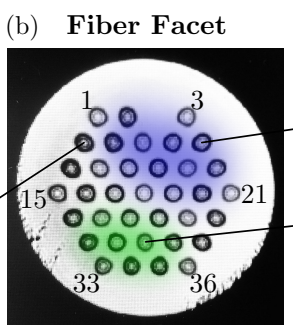

36 Core Few Mode Fiber

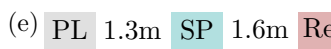

(e) $\mathrm{PL} 1.3 \mathrm{~m}$ SP $1.6 \mathrm{~m}$ Re

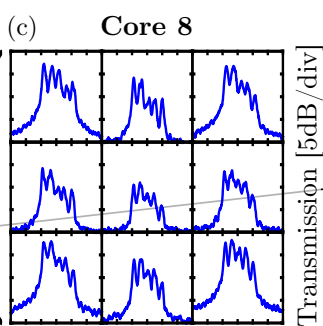

Time [20ps/div]

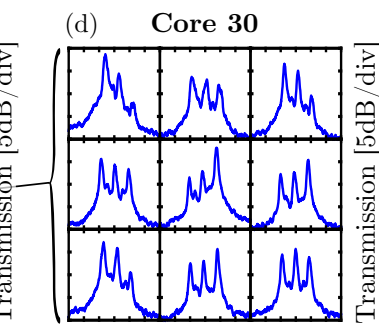

Time [20ps/div]

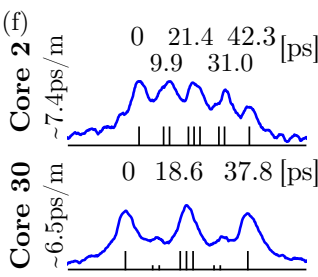

Fig. 2. Characterization results for 36-core few-mode photonic lantern. (a) Full $6 \times 6$ impulse response of the photonic lantern for core 4; (b) camera image of cleaved fiber facet, colored areas correspond to different behavior illustrated by: (c)-(d) reduced transfer matrices for cores 3 and 8. (e) Calculation of expected differential group delays between the two present mode groups; (f) example peak timings from cores 2 and 30.
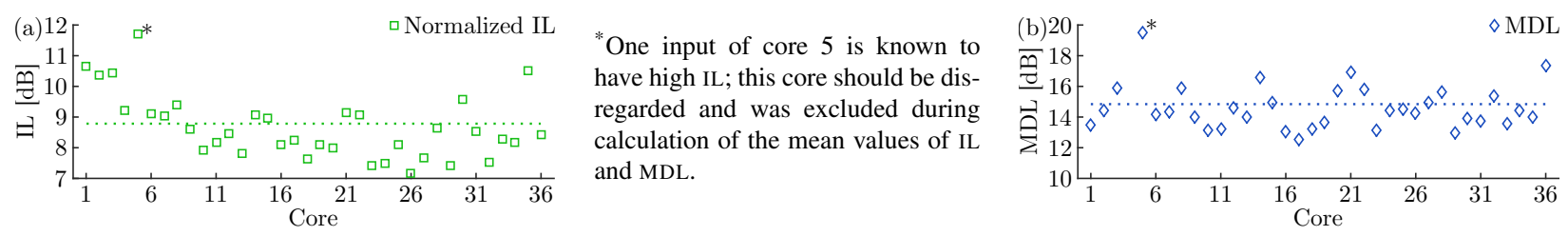

Fig. 3. Total system IL and MDL of all photonic lanterns, averaged from $1530 \mathrm{~nm}$ to $1570 \mathrm{~nm}$.

\section{Conclusions}

A fiber-coupled 36-core photonic lantern few-mode SMUX was characterized using an SDM-OVNA by measuring the complete impulse response of all 36 photonic lanterns, analyzing them in time domain and calculating IL and MDL. Further, analysis of mode-mixing within $2.9 \mathrm{~m}$ few-mode multi-core fiber coupled to the SMUX was performed, finding significant and core-dependent mode mixing at a splice present in the fiber.

Acknowledgment This work was partly funded by the DFF FTP mmW-SPRAWL project.

\section{References}

[1] W. Klaus et al., "Advanced space division multiplexing technologies for optical networks," J. Opt. Commun. Netw. 9, C1-C11 (2017).

[2] J. Sakaguchi et al., "Large spatial channel (36-core $\times 3$ mode) heterogeneous few-mode multicore fiber," J. Lightw. Technol. 34, 93-103 (2016).

[3] P. J. Winzer and G. J. Foschini, "MIMO capacities and outage probabilities in spatially multiplexed optical transport systems," Opt. Express 19, 16,680-16,696 (2011).

[4] N. K. Fontaine et al., "Characterization of space-division multiplexing systems using a swept-wavelength interferometer," in "Proc. OFC 2013," (2013), p. OW1K.2.

[5] P. Mitchell et al., "57 channel (19x3) spatial multiplexer fabricated using direct laser inscription," in "Proc. OFC 2014," (2014), p. M3K.5.

[6] D. K. Gifford, B. J. Soller, M. S. Wolfe, and M. E. Froggatt, "Optical vector network analyzer for single-scan measurements of loss, group delay, and polarization mode dispersion," Appl. Opt. 44, 7282-7286 (2005).

[7] G. VanWiggeren and D. Baney, "Swept-wavelength interferometric analysis of multiport components," IEEE Photon. Technol. Lett. 15, 1267-1269 (2003). 